This document is confidential and is proprietary to the American Chemical Society and its authors. Do not copy or disclose without written permission. If you have received this item in error, notify the sender and delete all copies.

\title{
The structural basis of small molecule targetability of monomeric Tau protein
}

\begin{tabular}{|r|l|}
\hline Journal: & ACS Chemical Neuroscience \\
\hline Manuscript ID & cn-2018-00182p.R1 \\
\hline Manuscript Type: & Article \\
\hline Domplete List of Authors: & $\begin{array}{r}\text { Kiss, Róbert; Hungarian Academy of Sciences, Research Centre for Natural } \\
\text { Sciences } \\
\text { Csizmadia, Georgina ; Research Center for Natural Sciences, Hungarian } \\
\text { Academy of Sciences } \\
\text { Solti, Katalin; Research Center for Natural Sciences, Hungarian Academy of } \\
\text { Sciences } \\
\text { Keresztes, Attila; Biological Research Center, Hungarian Academy of } \\
\text { Sciences, Biochemistry } \\
\text { Zhu, Max; Cantabio Pharmaceuticals } \\
\text { Pickhardt, Marcus; DZNE (German Center for Neurodegenerative } \\
\text { Diseases), } \\
\text { Mandelkow, Eckhard; Deutsches Zentrum fur Neurodegenerative } \\
\text { Erkrankungen } \\
\text { Toth, Gergely; University of Cambridge, Chemistry }\end{array}$ \\
\hline
\end{tabular}




\title{
The structural basis of small molecule targetability of monomeric Tau protein
}

\author{
Róbert Kiss, ${ }^{1}$ Georgina Csizmadia,${ }^{1 \dagger}$ Katalin Solti, ${ }^{1}$ Attila Keresztes, ${ }^{1 \uparrow}$ Max Zhu, ${ }^{2}$ Marcus Pickhardt, ${ }^{3,4}$ \\ Eckhard Mandelkow, ${ }^{3,4}$ Gergely Tóth ${ }^{1,2^{*}}$

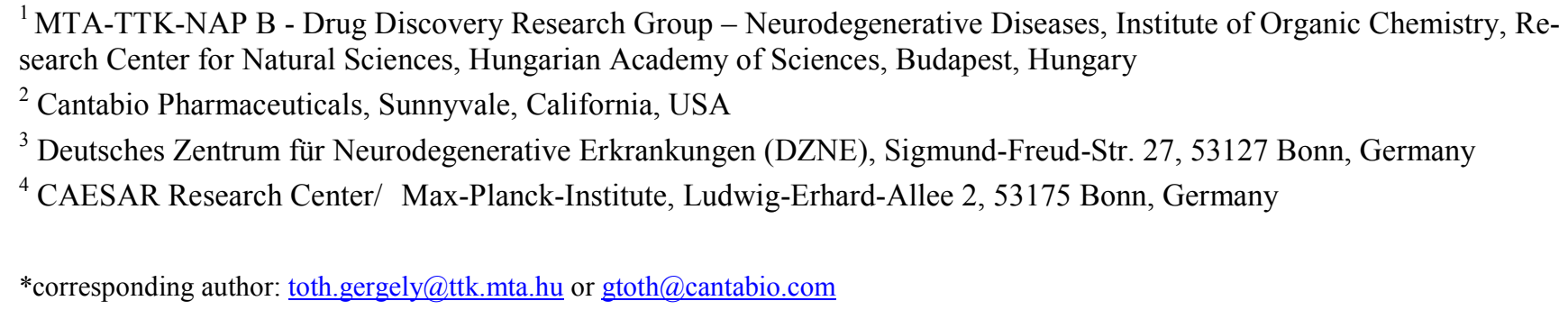

KEYWORDS: Tau, intrinsically disordered, modeling, conformational ensemble, methylene-blue, binding site, structure-based, drug design 


\begin{abstract}
The therapeutic targeting of intrinsically disordered proteins (IDPs) by small molecules has been a challenge due to their heterogeneous conformational ensembles. A potential therapeutic strategy to alleviate the aggregation of IDPs is to maintain them in their native monomeric state by small molecule binding. This study investigates the structural basis of small molecule druggability of native monomeric Tau whose aggregation is linked to the onset of Tauopathies such as Alzheimer's disease. Initially, two available monomeric conformational ensembles of a shorter Tau construct K18 (also termed Tau4RD) were analyzed which revealed striking structural differences between the two ensembles, while similar number of hot spots and small molecule binding sites were identified on monomeric Tau ensembles as on tertiary folded proteins of similar size. Remarkably, some critical fibril forming sequence regions of Tau (V306-K311, V275-K280) participated in hot spot formation with higher frequency compared to other regions. As an example of small molecule binding to monomeric Tau, it was shown that methylene blue (MB) bound to monomeric K18 and full-length Tau selectively with high affinity $\left(\mathrm{K}_{\mathrm{d}}=125.8 \mathrm{nM}\right.$ and $86.6 \mathrm{nM}$, respectively) with binding modes involving Cys291 and Cys322, previously reported to be oxidized in the presence of MB. Overall, our results provide structure-based evidence that Tau can be a viable drug target for small molecules and indicate that specific small molecules may be able to bind to monomeric Tau and influence the way in which the protein interacts among itself and with other proteins.
\end{abstract}




\section{INTRODUCTION}

Tauopathies $^{1}$ are a subset of neurodegenerative disorders, including Alzheimer's disease (AD) ${ }^{2}$ and frontotemporal dementias (FTD) ${ }^{3}$, which are linked to the accumulation of neurofibrillary tangles (NFTs) that are formed by the aggregation of microtubule associated protein Tau (MAPT) in patients' brain cells. The aggregation of monomeric Tau in neurons leads to the instability of microtubules in the axons, and the presence of Tau oligomers and fibrils generated through its aggregation cause toxic effects in neurons resulting in neurodegeneration.

Intrinsically disordered proteins (IDP) such as the Tau protein have been considered as intractable drug targets for small molecule drug discovery due to the lack of a stable 3D structure. Therefore Tau has been neglected by most small molecule drug discovery programs and has been mostly targeted by specific monoclonal antibodies for immunotherapy ${ }^{4-5}$. Since Tau is an intra-cellular protein localized mostly in neurons, it is not clear whether antibody-based drug development efforts will lead to efficacious treatments. Moreover, the economic cost of a potential immunotherapy for tens of millions of patients with Tauopathies will not likely be affordable for our society. Therefore, it is critical to explore alternative small molecule approaches targeting the Tau protein for the development of disease modifying therapeutics.

The soluble monomeric state of Tau has been studied by NMR spectroscopy ${ }^{6-9}$, which showed that the structure of monomeric Tau is made up of a diverse conformational ensemble. This conformational ensemble is not completely random: some pronounced intramolecular interactions exist and some regions have some propensity to adopt defined secondary structure ${ }^{6-9}$.

Computational structure-based modeling approaches are applied to generate conformational ensembles of IDPs, which use experimentally derived observations such as NMR, small-angle X-ray and FRET experimental data ${ }^{10}$. Two main approaches exist for generating conformational ensembles, the socalled pool-based and the molecular dynamics (MD) based approach. Typically, the extent and resolution of the experimental data is not sufficient for either approach to generate accurate high resolution 3D structures. Recently, two conformational ensembles of the monomeric form of Tau have been published $^{6,11}$.

We have previously explored whether small molecule binding pockets can exist within members of the heterogeneous conformational ensemble of IDPs despite their overall lack of persistent structures. Our results from investigating monomeric $\alpha$-synuclein ${ }^{12}$ and $A \beta 42$ peptide ${ }^{13}$ suggest that locally persistent binding sites are present even within a diverse population of conformations of these proteins. Moreover, 
a recent analysis of publicly available conformational ensembles of some IDPs found that IDPs have a comparable number of potential binding sites as proteins with ordered 3D tertiary structure ${ }^{14}$.

Binding of small molecules to such pockets may have distinct effects such as altering the conformational ensemble of IDPs and possibly impacting the dynamics and thermodynamic stability of the various conformational states ${ }^{12}$. The basis of the binding mechanism of small molecules to IDPs is yet to be clearly elucidated, nevertheless it has been assumed that it involves conformational selection by the binding ligand ${ }^{12-13,15}$. Consequently, targeting the conformational ensemble of the apo form of IDPs could be a viable structure-based drug discovery strategy. We previously successfully applied this approach for the basis of a structure-based computational docking screen on the conformational ensemble of $\alpha$-synuclein to identify novel fragment and drug-like small molecules targeting monomeric $\alpha$ synuclein, one of which was active in cellular models of $\alpha$-synuclein-mediated dysfunction ${ }^{12}$. In another recent study, novel compounds binding to c-Myc were identified by structure-based virtual screening on three binding pockets of representative c-Myc conformations ${ }^{16}$.

Only a limited number of small molecule ligands of monomeric Tau have been described in the literature $^{17-18}$. Most of the identified compounds are, however, suboptimal for further drug discovery optimization, as they have either non-druglike physicochemical properties or contain reactive functional groups that are frequently associated with toxicity issues and lack of specificity ${ }^{19-21}$. Recently, we applied a unique biophysics based binding screening methodology to detect the binding between small molecules and monomeric Tau, which led to the identification of a diverse set of novel fragment and lead-like small molecules capable of interacting with Tau, some of which were able to reduce the aggregation of Tau in vitro and in a cellular model of Tauopathies ${ }^{22}$. The study demonstrates that monomeric Tau can be a viable receptor of small drug-like molecules, and supports the potential and practical feasibility of the therapeutic strategy to target early phases of the aggregation pathway of Tau and potentially other IDPs by small molecules, thereby eliminating the formation of potential toxic misfolded protein species. This and other studies suggest that the structure-based identification of novel small molecules binding to IDPs, such as monomer Tau, could be a viable drug discovery strategy ${ }^{23}$.

One of the most studied Tau interacting ligand is methylene blue (MB) (Fig. S1), an approved treatment for methemoglobinemia, which has been shown to have a wide range of interacting protein partners and biological activities ${ }^{24}$. MB was shown to inhibit Tau aggregation ${ }^{25}$ and to disintegrate Tau fibrillar aggregates. In particular, Taniguchi et al. showed that MB inhibits heparin-induced Tau filament formation $\left(\mathrm{IC}_{50}=1.9 \mu \mathrm{M}\right)^{26}$. Thionine (a desmethyl derivative of MB) showed inhibition of Tau-Tau binding $\left(\mathrm{IC}_{50}=98 \mathrm{nM}\right)^{25}$. MB also promoted the oxidation of the cysteine residues of Tau (C291 and $\mathrm{C} 322)$, to sulfenic (SO), sulfinic $\left(\mathrm{SO}_{2}\right)$ and sulfonic $\left(\mathrm{SO}_{3}\right)$ forms, in vitro, and thus presumably inhibit 
the aggregation of the protein by retaining it in an oxidized monomeric conformation ${ }^{27-28}$. Interestingly, MB also inhibited caspase 1 and 3 activities in vitro through the oxidization of their catalytic cysteine ${ }^{29}$. Moreover, MB protected from Tau over-expression caused neurotoxicity in vivo ${ }^{30-31}$ and MB based therapeutic candidate, LMTX ${ }^{\circledR}$ (TauRx's second-generation Tau aggregation inhibitor (TAI)), has been through several phase 3 clinical trials in $\mathrm{AD}$ and is still under investigation in further phase 3 clinical trials in $\mathrm{AD}$.

We set out to continue to investigate the small molecule targetability of IDPs, by focusing on Tau protein. Small molecules may have the potential to bind to specific conformations of monomeric Tau that could influence the way in which Tau interacts among itself and with other proteins in neurons. Thus, binding small molecules to monomeric Tau could alter its aggregation propensity, or perturb the malfunction or the toxicity in neurons caused by Tau aggregation, or influence Tau modulated signal transduction. In this study, we characterized the structural diversity and availability of small molecule binding sites within two published conformational ensembles of Tau and analyzed their applicability to rational drug design. Moreover, we investigated binding ability of MB to monomeric Tau as a model system for Tau interactions using computational structure based and biophysical experimental approaches. Our results yield further insights on the applicability of available IDP conformational ensembles, existence of binding sites, and provide a better understanding of the targetability of monomeric Tau by small molecules.

\section{RESULTS}

\section{Characterization of monomeric Tau ensembles}

The two publicly available 3D structural ensembles of monomeric K18 Tau, comprising the repeat domain responsible for aggregation, were collected and analyzed to assess their structural diversity and their potential applicability for structure-based drug discovery. Ensemble 1 (ENS\#1) was reported by Ozenne et. al. ${ }^{6}$ consisting of 801 Tau conformers at high resolution, and the second ensemble 2 (ENS\#2) was reported by Fisher et. al. ${ }^{11}$ containing 75 conformers.

Both Tau ensembles were found to be substantially diverse because the closest internal conformers had a root-mean-square deviation (RMSD) of $11.28 \AA$ (ENS\#1) and $9.26 \AA$ (ENS\#2), respectively. The overall diversity of the individual ensembles was similar when all RMSD matrix elements were considered (average RMSD) (Fig. 1A), while the minimum RMSD of each conformer to its closest neighbor (minimum RMSD) was larger for ENS\#2 (Fig. 1B), which may be attributed to the lower ensemble size of ENS\#2. 

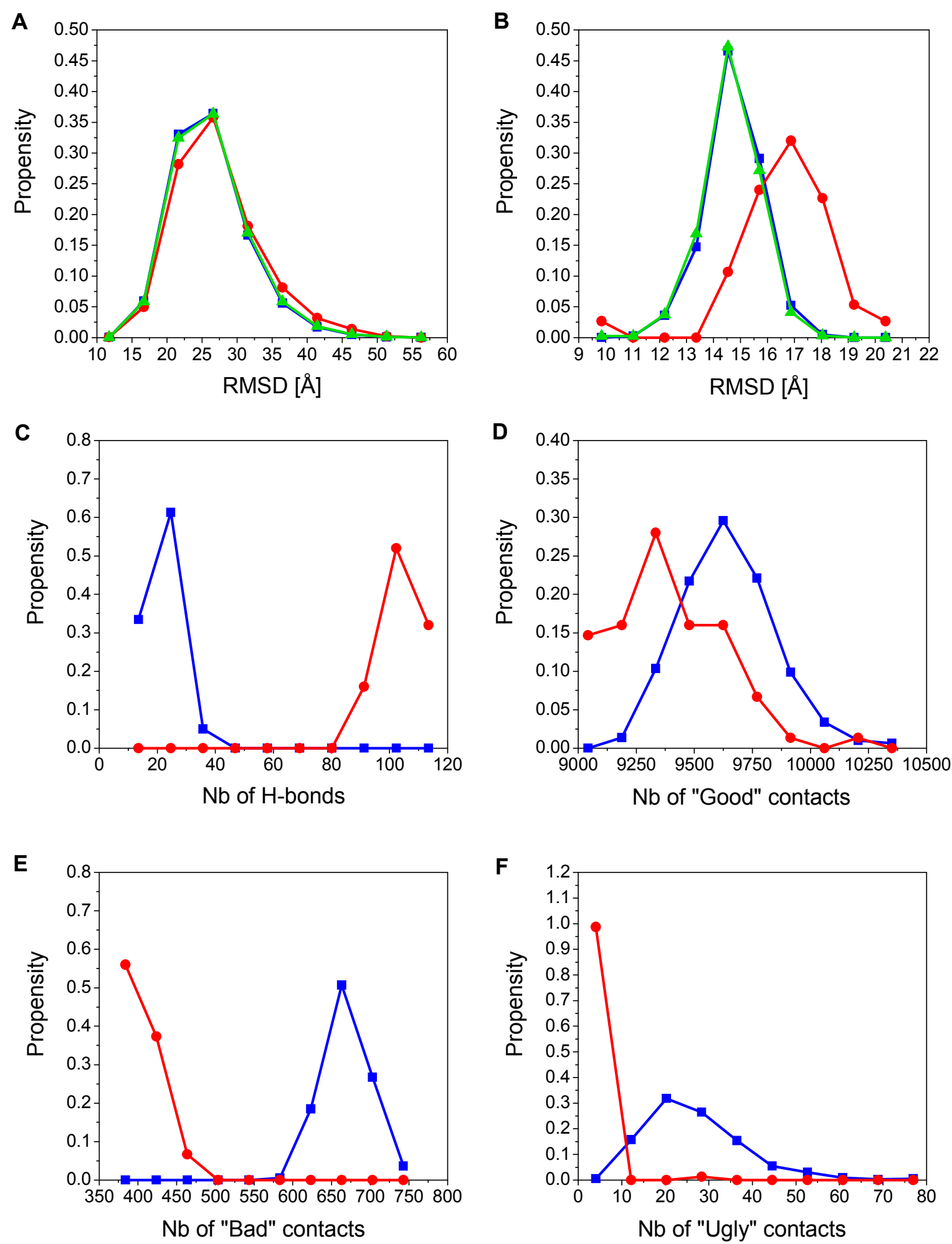

Figure 1. RMSD distribution curves of monomeric Tau ensembles ENS\#1 (blue), ENS\#2 (red) and the combined ensemble (green) calculated for all RMSD matrix elements (average RMSD) (A) and between each conformer and their closest conformer (minimum RMSD) (B). Distribution of the number $(\mathrm{Nb})$ of intramolecular contacts in monomeric Tau ensembles ENS\#1 (blue) and ENS\#2 (red): number of H- 
bonds (C), number of "Good" contacts (D), number of "Bad" (E) and number of "Ugly" (F) contacts (see definitions in Methods).

Surprisingly, when the two ensembles were combined, the closest conformers in the combined set had an RMSD of $9.26 \AA$ (as in ENS\#2) exposing that the two ensembles contain significantly different conformations. (The full RMSD matrices are shown in Fig. S2). To assess the compactness of the conformers, intramolecular contacts were calculated. Interestingly, many more van der Waals contacts ("Good", "Bad" and "Ugly" - see definitions in Methods) were found in ENS\#1, while ENS\#2 contained more Hbonds (Fig. 1C-F). To further assess the compactness of the ensembles, the radii of gyration (Rg) of the conformers were calculated. ENS\#1 had lower Rg value distribution in line with the higher number of van der Waals contacts (Fig. S3). The secondary structural content of the conformers was calculated, which indicated that some regions show higher propensity of helical and $\beta$-sheet secondary structures (Fig. 2A and 2B).

A
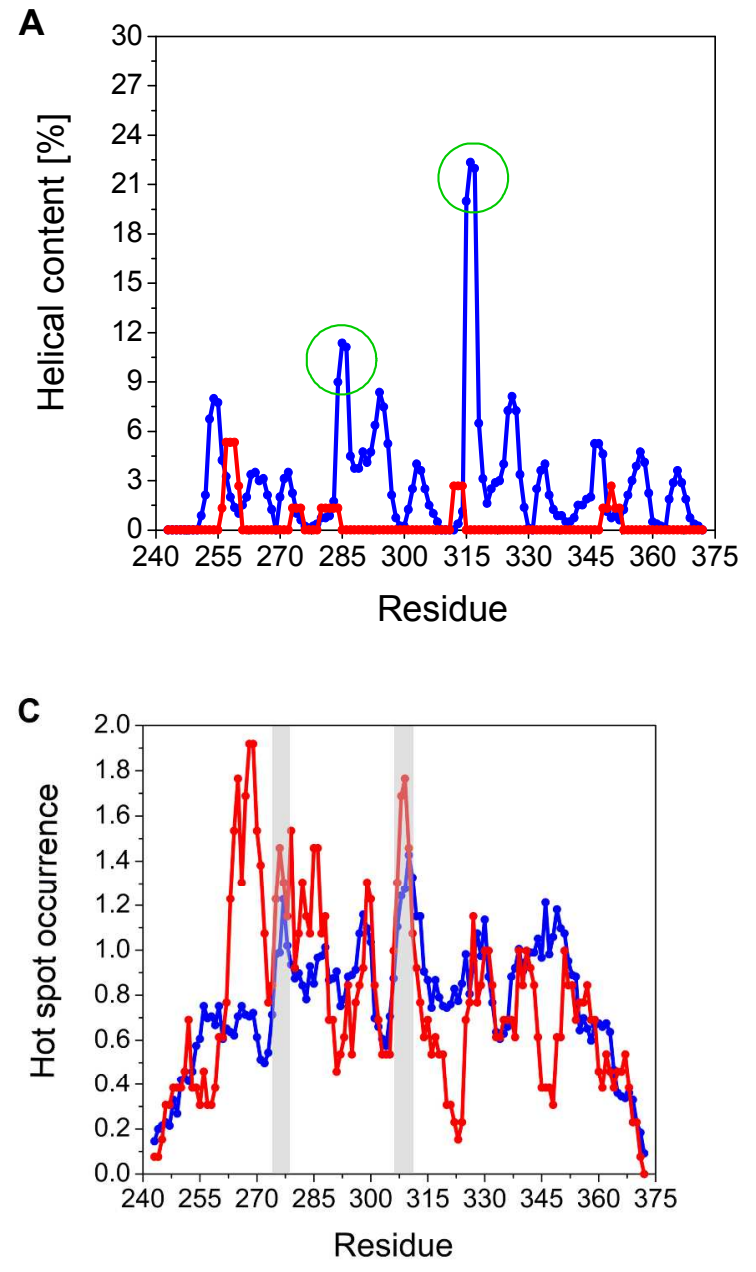

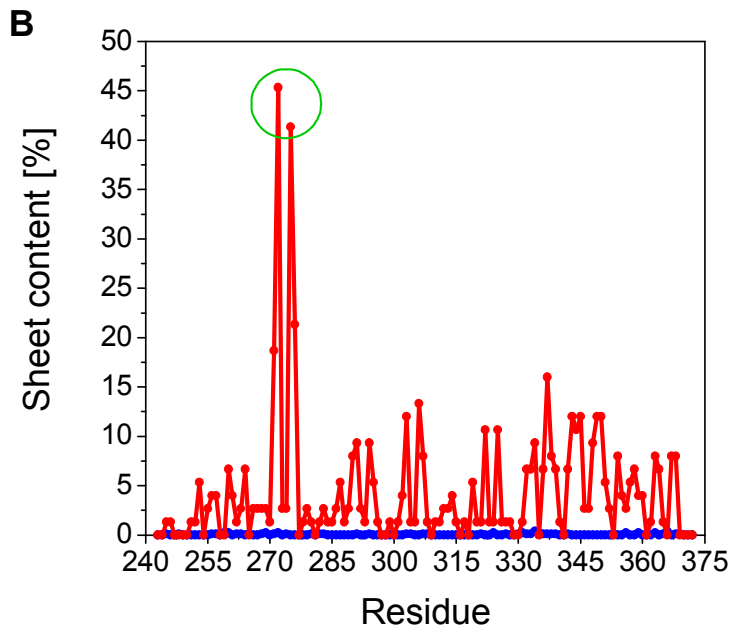

Residue 
Figure 2. Distribution of helical (A) and $\beta$-sheet (B) secondary structure content in monomeric Tau ensembles ENS\#1 (blue) and ENS\#2 (red). Regions of higher secondary structure propensity are marked by green circles. Hot spot formation propensity $(\mathrm{C})$ of residues in monomeric Tau ensembles ENS\#1 (blue) and ENS\#2 (red). Fibril-forming regions (V306-K311 and V275-K280) are indicated by larger markers / dashed lines.

In particular, the secondary structure analysis suggested higher propensity for nascent helices between residues L284-N286 and L315-K317 and increased propensity for $\beta$-structured conformation between residues G271-Q276. This latter region coincides with the V275-K280 sequence that has been shown to play a critical role in forming the core of Tau fibrils ${ }^{32-34}$. Moreover, the P270-G273 region has been suggested to form a $\beta$-hairpin conformation ${ }^{35}$. Unexpectedly, the two ensembles feature completely different secondary structure elements: helical structures were solely found in ENS\#1, while $\beta$ structures were only present in ENS\#2 (Fig. 2A and 2B).

\section{Small molecule binding site mapping}

Next, all Tau conformers were mapped for the presence of potential hot spots and small molecule binding sites using FTMap ${ }^{36}$. The mapping results revealed that the intrinsically disordered monomeric state of Tau contained similar number of binding hot spots (Fig. 3A and 3B) and binding sites (Fig. 3C) as the investigated folded proteins with tertiary structure.
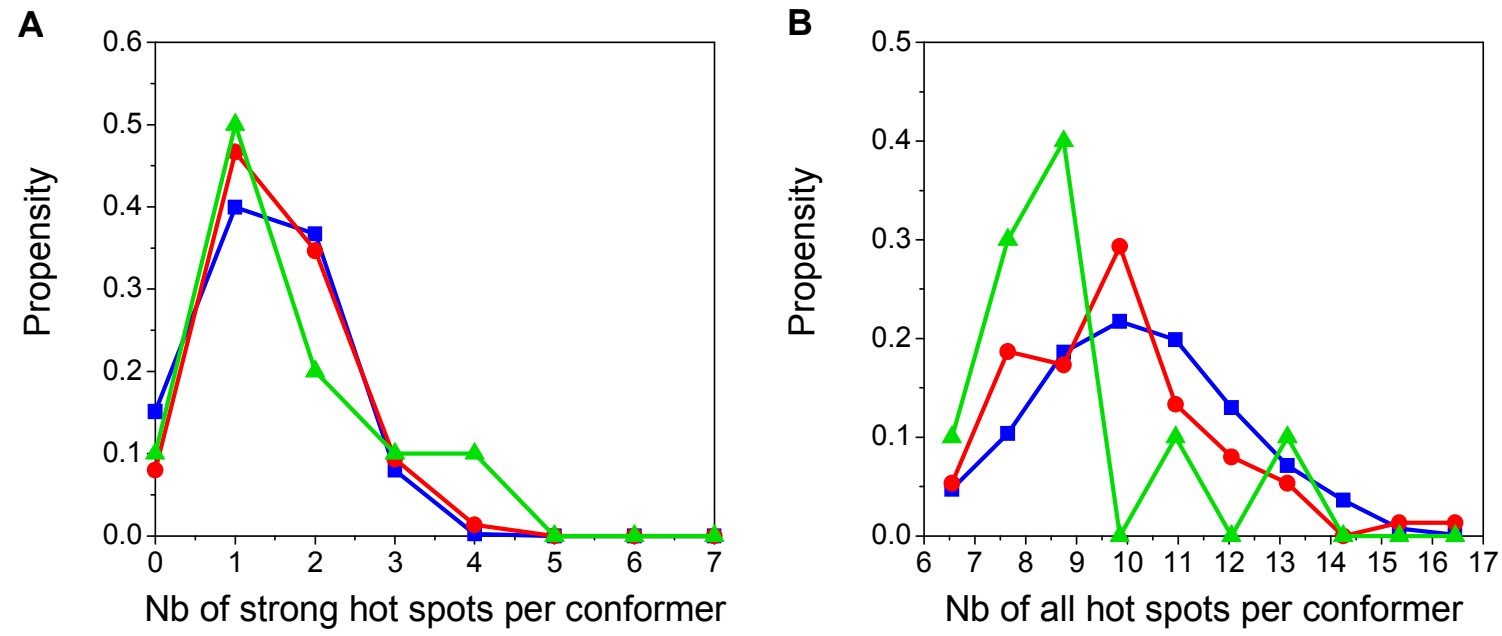

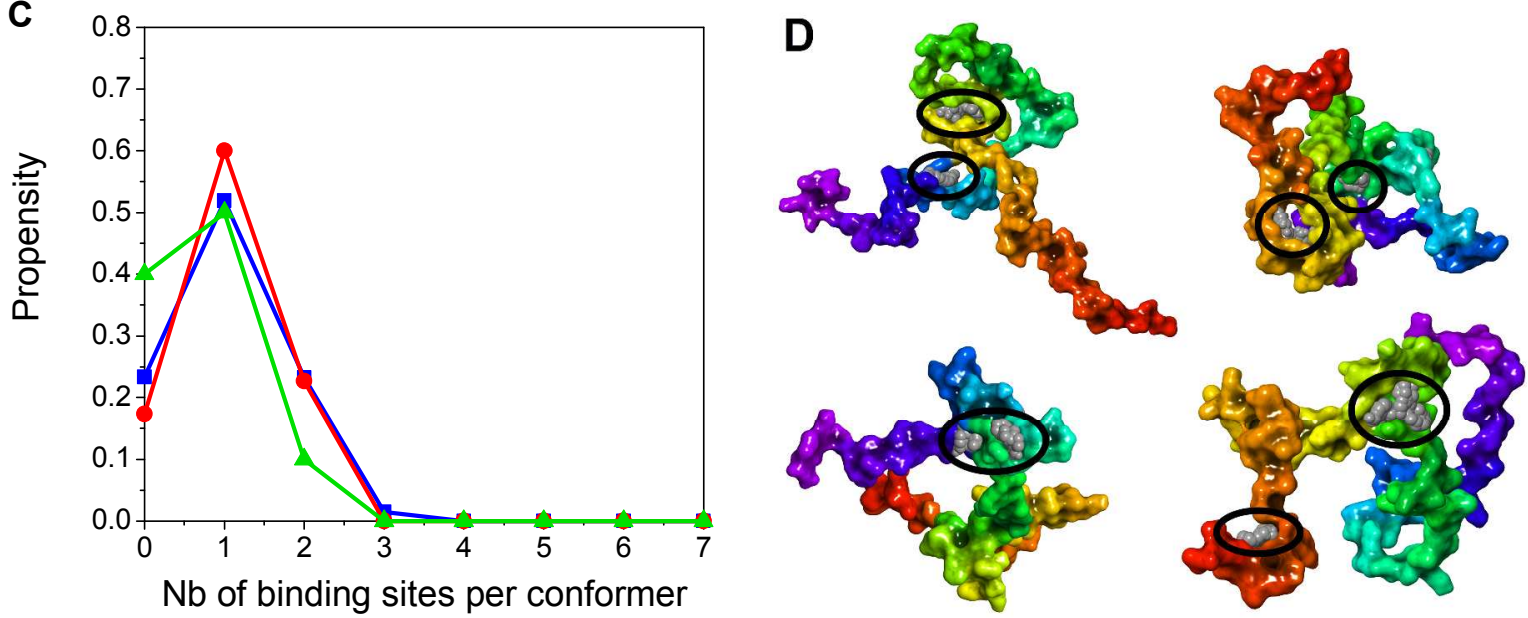

Figure 3. Distribution of the number $(\mathrm{Nb})$ of strong binding hot spots $(\mathrm{A})$, number $(\mathrm{Nb})$ of all binding hot spots (B) and binding sites (C) in monomeric Tau ensembles ENS\#1 (blue) and ENS\#2 (red) and folded proteins (green) (see definitions of strong hot spots and binding sites in Methods). Binding hot spot examples (black circles) on selected Tau conformations (D).

In particular, the distribution of strong hot spots (see definition in Methods) that are essential for binding site formation was almost identical for ENS\#1 and ENS\#2 as for folded proteins (Fig. 3A). Moreover, the Tau ensembles contained more weak hot spots and an approximately similar number of binding sites compared to folded tertiary proteins (Fig. 3). Solvent accessible surface area (SASA) of the hot spots showed similar distribution for ENS\#1 and ENS\#2 (Fig. S4).

The tendency of individual residues to form binding hot spots was also analyzed. The two most critical fibril forming regions of Tau, the hexapeptide motifs V306-K311, V275-K280, are among the most frequently occurring residues in forming the hot spots (Fig. 2C). In particular, the V306-K311 region formed hot spots with highest propensity in ENS\#1 and second highest in ENS\#2. These results suggest that the monomeric structural ensembles of Tau investigated contain hot spots and targetable small molecule binding sites, which indicates that intrinsically disordered Tau may be a tractable target for small molecule drug discovery.

\section{Investigation of the monomeric Tau binding characteristics of methylene blue}

To elucidate further the interaction between MB with Tau, we investigated whether MB can oxidize cysteines in general by using glutathione (GSH), as standard model molecule generally used in covalent drug discovery ${ }^{37}$ with the aim to gain insights into the thiol oxidation reactivity of MB. Our experiments 
showed that MB does not form any covalent complexes with GSH, however, it facilitates the oxidation of GSH (Fig. 4A).
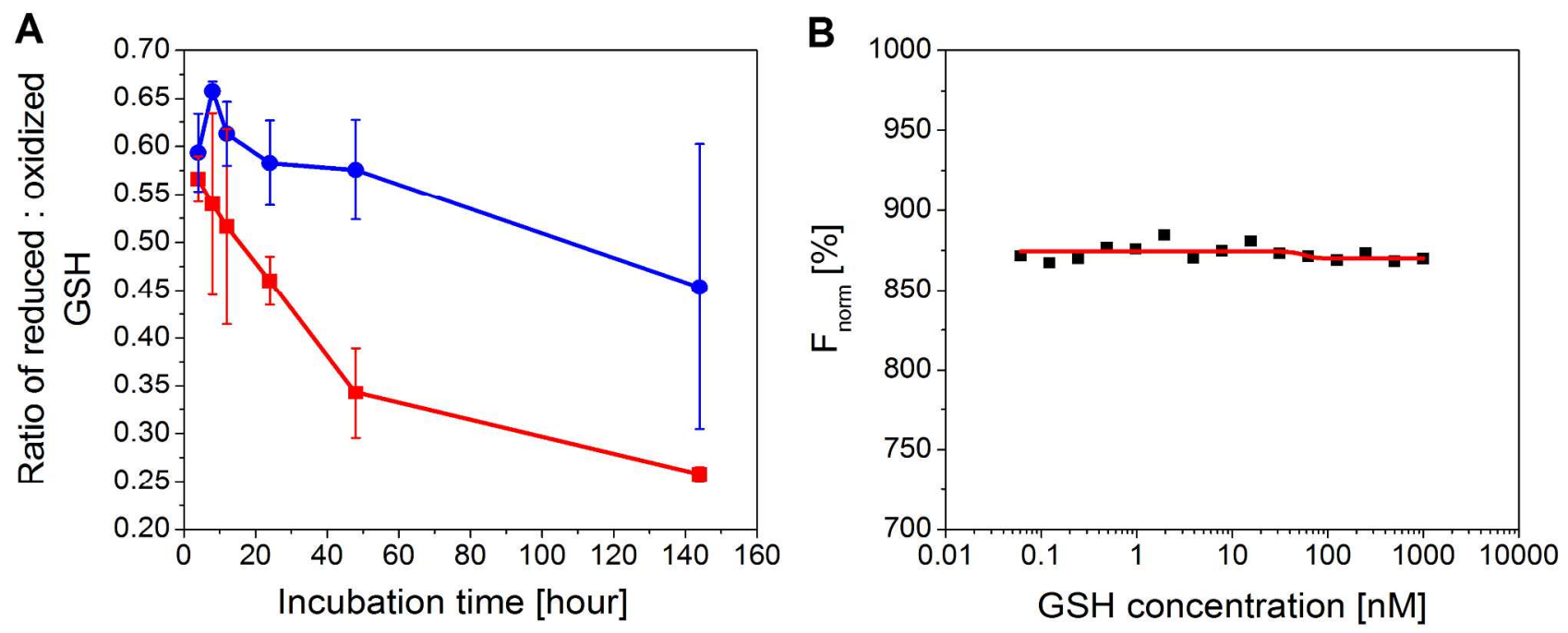

Figure 4. Oxidation kinetics of glutathione (GSH) in the presence (red) and absence (blue) of methylene blue (MB) monitored by LC-MS (A). Standard deviation is indicated by error bars. Dose-response curve between GSH and MB based on measured microscale-thermophoresis shows that no direct interaction could be detected (B). The fluorescence of MB was exploited for the MST experiments.

Given that GSH is a small flexible peptide, it is unlikely that MB has a specific affinity to GSH driven by non-bonded interactions. To elucidate this we tested whether GSH binds to MB using microscale thermophoresis (MST). The strong fluorescence properties of MB (Exc $\max _{\text {ax }}: 665 \mathrm{~nm}$; $\left.\mathrm{Em}_{\max }: 692 \mathrm{~nm}\right)$ enables its label-free application as a sensitive molecular interaction detection system in MST. The MST experiments showed no sign of the existence of detectable interaction between MB and GSH (Fig. 4B). This suggests that the oxidation of GSH by MB was driven by diffusional effects.

Next, this MST set-up was further applied to characterize the affinity of MB to monomeric Tau. The concentration-dependent change in thermophoresis and T-jump signals indicated that MB binds both to monomeric full-length (Fig. 5A) and to monomeric K18 Tau with $\mathrm{K}_{\mathrm{d}}$ values of $86.6 \pm 8.8 \mathrm{nM}$ and 125.8 $\pm 5.4 \mathrm{nM}$ (Fig. 5B), respectively, whereas MB bound only weakly to BSA $\left(\mathrm{K}_{\mathrm{d}}>1 \mu \mathrm{M}\right)($ Fig. 5C). 


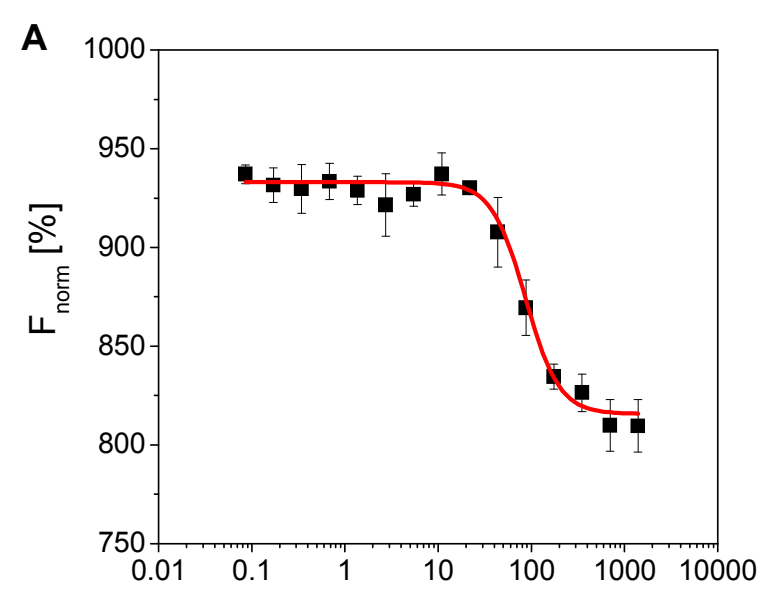

Full length Tau concentration [nM]

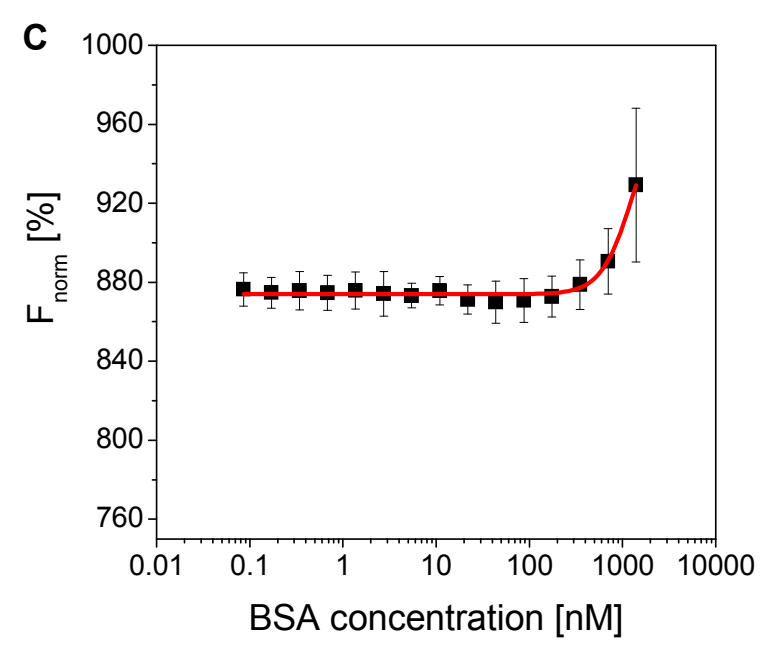

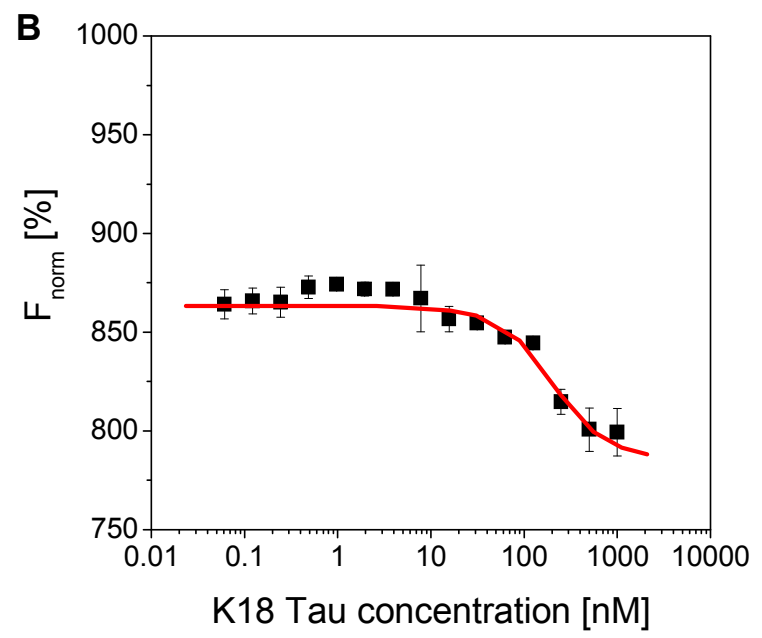

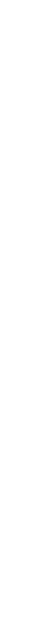

Figure 5. Dose-response curve between MB and monomeric full-length Tau (A), K18 Tau (B) and bovine serum albumin $(\mathrm{C})$ based on measured combined $\mathrm{T}_{\text {jump }}$ and microscale thermophoresis data.

Overall, these results suggest that MB binds to monomeric Tau with reasonably high affinity and with some specificity, and this binding is non-covalent by nature. Consequently, MB herein will be used as a model ligand to evaluate the targetability of monomeric Tau ensembles by structure-based docking of small molecules.

\section{Exploration of the binding site of methylene blue to monomeric Tau}

To identify the potential binding sites of MB to monomeric Tau, we applied structure-based docking calculations to each of the predicted small molecule binding sites of the Tau conformations from both ensembles. All possible binding modes of MB generated by the docking program FRED were rank ordered by a consensus scoring scheme (see Methods for details), which provided a rank of the predicted binding modes of MB to Tau. The distribution of the consensus ranks is plotted in Fig. 6 . 


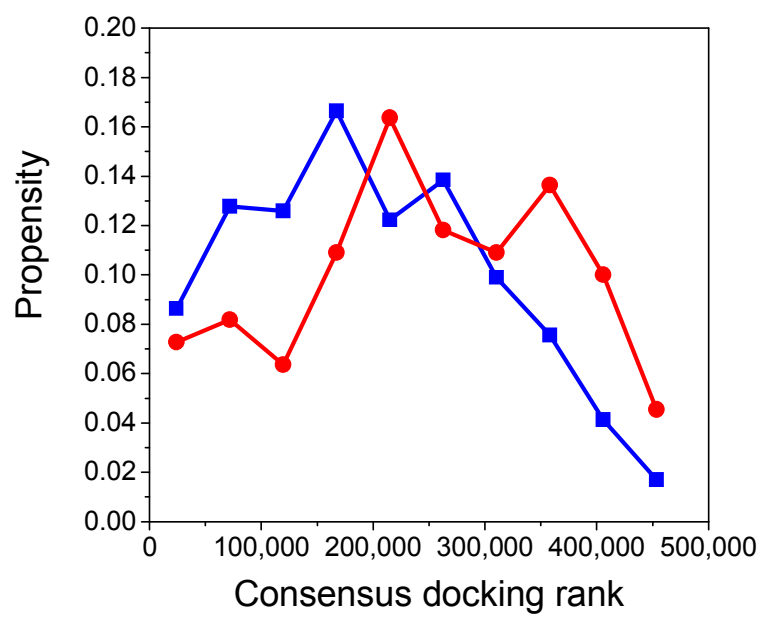

Figure 6. Distributions of the ranks of MB binding modes to the binding sites of monomeric Tau ensembles ENS\#1 (blue) and ENS\#2 (red).

Several potential binding modes of MB at different binding sites of monomeric Tau were identified in both Tau ensembles. The docking results indicated more favorable binding of MB to Tau conformations in ENS\#1 compared to ENS\#2 (Fig. 6). Binding conformations in close proximity of C291 and C322 were selected as these residues were suggested to be directly involved in MB binding on the basis of NMR HSQC measurements and the oxidation of both cysteine residues due to $\mathrm{MB}^{28}$. $206 \mathrm{MB}$ binding poses were identified, where either C291 or C322 was found within $6 \AA$ proximity of MB. Out of these 206 binding poses, 9 (8.0\% hit rate) belonged to ENS\#1 and 197 (17.7\% hit rate) to ENS\#2, respectively (Fig. S5).

These MB binding modes were ranked by the consensus docking ranks and the highest ranked $10 \%$ (21) of the poses were selected for further analysis. In the 21 selected poses ENS\#2 was overrepresented: 20 poses (1.8\%) from ENS\#2 compared to 1 pose $(0.9 \%)$ from ENS\#1. In one of the 21 finally selected docking poses MB was found within $6 \AA$ from both C291 and C322 (Fig. 7A and 7B). 
$\mathbf{A}$

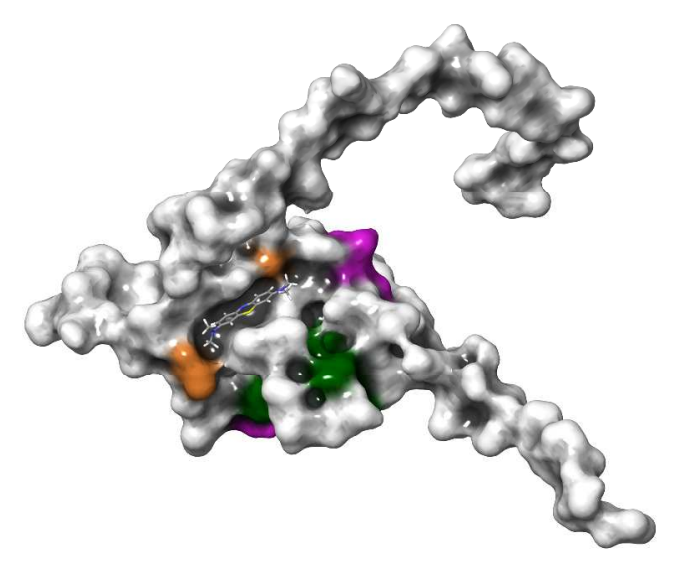

B

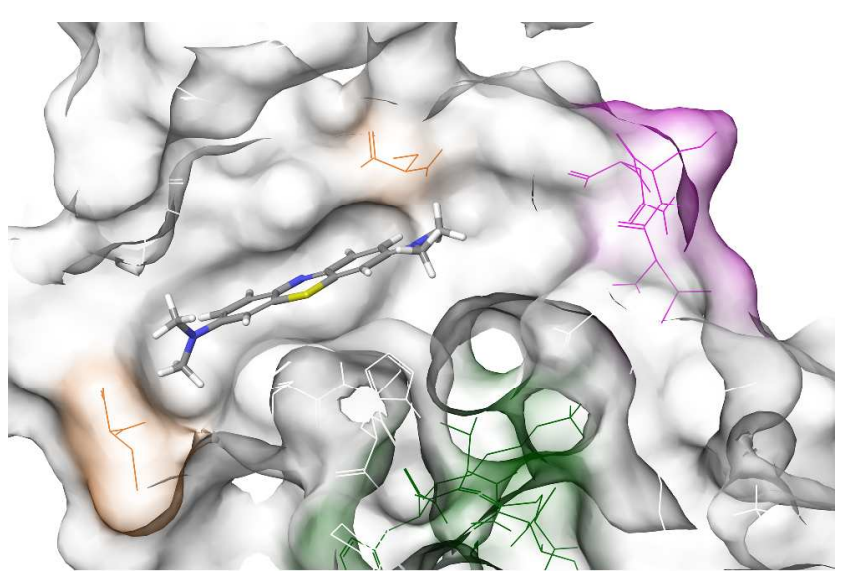

C

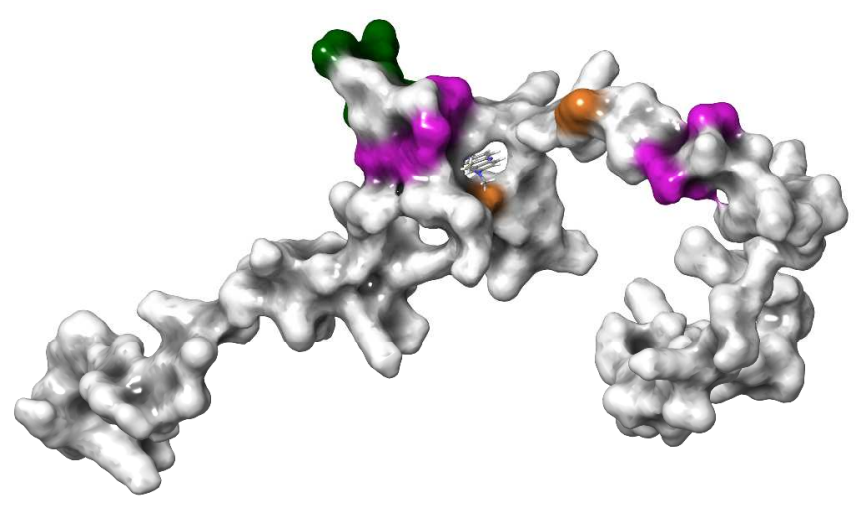

D

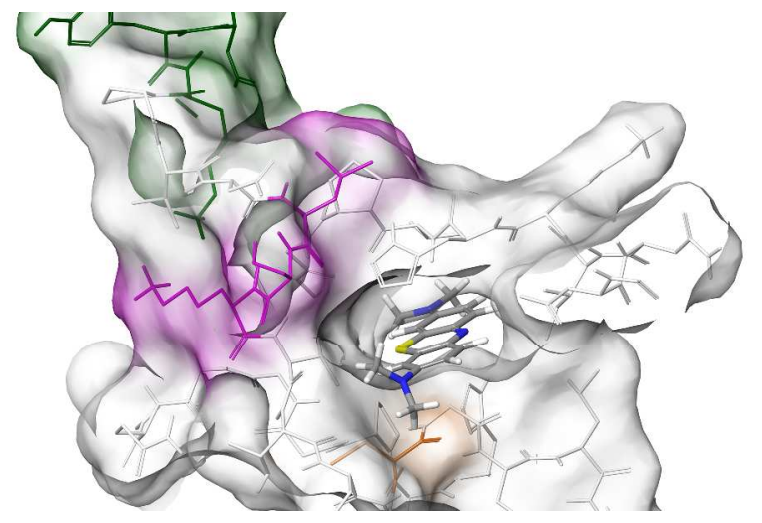

Figure 7. Predicted binding poses of MB in selected representative conformations of K18 of Tau. Binding pose \#4 (A, B): MB is in close proximity to both C291 and C322. Binding pose \#1 (C, D): highest ranked binding pose of MB by consensus scoring (Coloring scheme is the following: C291 and C322 in orange, fibril forming region V306-K311 in green and nascence helical regions L284-N286 and L315$\mathrm{K} 317$ in magenta).

The highest ranked binding mode of $\mathrm{MB}$ is shown in Fig. 7C and 7D. In both binding modes the fibril forming V306-K311 region as well as the nascent helical regions (L284-N286 and L315-K317) contributed to binding site formation. All interacting residues are listed in Table S1. 
In summary, we identified potential binding sites and modes of MB to Tau, using previous experimental results ${ }^{28}$ demonstrating that structure-based modeling can be applied on conformational ensembles of monomeric Tau. This suggests that such approach may be also useful for the development of Tau targeting small molecule therapeutic candidates.

\section{DISCUSSION AND CONCLUSION}

\section{Comparison of two distinct monomer Tau conformational ensembles}

While both Tau conformational ensembles analyzed satisfy the global experimental parameters of monomeric Tau according to their authors, striking structural differences were identified between the two ensembles. In particular, the closest conformers between ENS\#1 and ENS\#2 were more dissimilar than the closest conformers in ENS\#2 (RMSD = 9.26 $\AA$ ). In other words, the conformations of the two ensembles had significantly different RMSD at the global level, although this does not exclude the possibility of local similarities. In line with the RMSD differences, the internal contact profiles of the two ensembles also differed substantially. Moreover, nascent helical and $\beta$-sheet structures were unevenly distributed in the two ensembles: helical structures were solely found in ENS\#1, while $\beta$-sheet structured conformations were only captured by ENS\#2.

Plausible explanations for these striking differences in the two ensembles may be the followings. The level of disorder in monomeric Tau has been particularly high and the acquisition of relevant experimental data that can be translated to structural constraints is limited in extent and resolution. Therefore multiple possible conformational ensembles can be generated that satisfy the limited number of experimental constraints. The sampling of both reported ensembles may be insufficient, as they are selected to satisfy all constraints by the smallest possible set of conformers. In addition, more, relevant experimental data that can be translated to local structural constraints and utilized during ensemble generation are needed. In particular, it has been suggested that novel techniques capable of sampling proteins on a longer timescale would be required for a better characterization of $\operatorname{IDPs}^{38}$.

Nevertheless, in both cases, the individual conformations of the published ensembles may represent relevant, energetically favored conformations of monomeric Tau that can be targeted by structure-based drug discovery. The results of this study suggest that the folding of different segments of Tau is required for the formation of the binding site and the complete monomeric Tau structure is not required for accommodating small molecule ligands. We also made similar observation when studying the conformational ensemble of intrinsically disordered $\alpha$-synuclein ${ }^{12}$ and $A \beta 42^{13}$. Similarly to the $\alpha$-synuclein study $^{12}$, we identified similar number of hot spots and binding sites on the ensembles compared to ran- 
domly selected folded proteins possessing tertiary structure suggesting that it is feasible to identify high affinity ligands to Tau and that this may be a general character of IDPs.

Remarkably, we found that some critical fibril forming regions (V306-K311, V275-K280) of Tau participated in hot spot formation with higher frequency compared to other sequence regions. These regions may be particularly relevant when targeting Tau with small molecules as they could potentially block fibril formation by competing with other Tau interacting surfaces that are critical for aggregation.

\section{On the binding mechanism of MB to Tau}

To demonstrate the applicability of the available conformational ensembles of Tau for structure-based drug discovery, we selected MB and predicted its potential binding mode to Tau. To establish the MB Tau complex as a validated model system for this study, we further explored its mechanism of interaction between $\mathrm{MB}$ and monomeric Tau in vitro. MB has been shown to bind to monomeric Tau previous$1 y^{28}$.

The interaction of MB with monomeric Tau is complex. MB has been shown to be able to oxidize cysteine residues of monomeric Tau ${ }^{27-28}$. However, this is likely a non-specific event, as we show that MB can oxidize solvent accessible cysteines on a small molecule as GSH. Nevertheless, we observed concentration-dependent change in thermophoresis of MB when either full-length or K18 Tau was administered. In contrast, no such changes were observed in the thermophoresis of MB when GSH was administered to it instead of Tau. This suggests that MB binds to monomeric Tau reversibly via nonbonded interactions. The docking simulations of MB revealed potential binding modes that are in reasonably good agreement with the available experimental data. In particular MB was found to bind in close proximity of C291 / C322 which were shown to be oxidized during MB administration ${ }^{28}$.

\section{Small molecule binding sites exist on monomeric Tau}

Our study of monomeric Tau structural conformational ensembles supports the results of our previous theoretical studies on $\alpha$-synuclein ${ }^{12}$ and $\mathrm{A} \beta 42^{13}$ ensembles in regard to the existence of small molecule binding sites on these IDPs. Our results in this study on the mechanism of binding of MB to Tau provide further experimental and theoretical evidence for the existence of bindings sites on Tau. Additional evidence of this was demonstrated by our previous study ${ }^{22}$ in which we applied a biophysics-based high throughput screen to identify small molecule binders to monomeric full length Tau. A diverse set of novel fragment and lead-like small molecules were capable of binding monomeric Tau, some of which had also the ability to inhibit Tau aggregation. Overall, these results demonstrate that despite its heterogeneous conformational ensemble and a lack of stable tertiary structure, Tau can be a viable receptor for 
small drug-like molecules. These findings have strong relevance to other IDPs in general and imply that other IDPs may also have the ability to interact with drug-like small molecules. Our results imply that specific small molecules may exist that could bind to monomeric Tau and influence the way in which Tau interacts among themselves and with other proteins in neurons. These small molecules therefore could alter the aggregation propensity of Tau and/or perturb Tau mediated cellular signal transduction.

\section{METHODS}

\section{Structural data sources}

Conformational ensembles of monomeric K18 Tau were collected from the PED database (http://pedb.vib.be/) ${ }^{39}$ (ENS\#1) and from the supplementary material of the research article of Fisher et al. (http://www.rle.mit.edu/cbg/data.htm) ${ }^{11}$ (ENS\#2). Initial ENS\#1 ensemble contained 995 structures. After superposition and backbone RMSD calculations 194 duplicate structures were eliminated resulting in 801 structures (referred as ENS\#1 throughout the manuscript). ENS\#2 contained 75 individual structures that represents conformations with at least 0.0005 calculated probability. All 75 structures were found unique after superposition and backbone RMSD calculations.

X-ray structures of proteins with identical length of K18 Tau (130 residues), with a maximum 30\% sequence identity were selected from the PDB database [http://www.rcsb.org]. Ten (PDB IDs: 1KNM, 2NWD, 3FYM, 3RFE, 4ESP, 4HWM, 4PS6, 4XPX, 4YTK, 5C6S) out of the 53 resulting structures were selected based on the resolution of the structures.

\section{RMSD matrix calculations}

Tau structures were superimposed by their C-alpha atoms and backbone RMSD matrices were calculated for both ensembles in Maestro ${ }^{40}$ using Conformer Cluster.

\section{Prediction of side chain conformations}

Original version of ensemble ENS\#1 lacked side chain conformations. RASP ${ }^{41}$ was used by default parameters to calculate the missing side chains for ENS\#1.

\section{Molecular contacts calculation}

Intramolecular contacts were calculated and classified in Maestro ${ }^{40}$ as follows: H-bonds: H...A distance must be less than $2.8 \AA$, the D-H...A angle must be greater than $120^{\circ}$, and the H...A-B angle must be greater than $90^{\circ}$.

Contacts are defined by a ratio given by the following formula: 


$$
\mathrm{C}=\mathrm{D} 12 /(\mathrm{R} 1+\mathrm{R} 2)
$$

where D12 is the distance between atoms 1 and 2, and R1 and R2 are the van der Waals radii of atoms 1 and 2. The contacts are classified into "Good", "Bad", and "Ugly" based on this ratio. The default values are: Good: $1.30>\mathrm{C}>0.89$; Bad: $0.89>\mathrm{C}>0.75$; Ugly: $\mathrm{C}<0.75$.

\section{Secondary structure calculation}

Per residue secondary structure was determined for all conformations of monomeric Tau by DSSP (http://swift.cmbi.ru.nl/gv/dssp) ${ }^{42}$ with default parameters.

\section{Binding site mapping}

The FTMap web server ${ }^{36}$ (http://ftmap.bu.edu/) was used to identify binding hot spots and binding sites on the Tau conformers and selected folded proteins. According to the authors" instructions " 43 "strong hot spot" was defined as a hot spot with a cross-cluster population $>16$. Additionally, a "binding site" was defined as a minimum set of two hot spots (one of which is a strong hot spot) within $8 \AA$ distance.

Solvent accessible surface area of each hot spot was calculated in Maestro ${ }^{40}$ using Create Binding Site Surfaces panel including residues within $5 \AA$ of a single cross-cluster (set of docked solvent molecules by FTMap).

\section{Ligand preparation}

$3 \mathrm{D}$ conformations of methylene blue (MB) were calculated by OMEGA ${ }^{44-45}$ by applying more thorough settings than default (strictfrags (true), sampleHydrogens (true), ewindow (20.0), maxconfs $(10,000)$, maxtime (600.0), rms (0.3)). QUACPAC ${ }^{46}$ was used to calculate am1bcc charges for each conformer (option: am1bccsym).

\section{Molecular docking}

Tau conformations were prepared for docking by using the pdb2receptor function of OEDocking ${ }^{47-50}$. Binding sites were defined based on the cross-clusters (set of docked solvent molecules by FTMap). Docking was carried out by FRED $^{51}$ applying special parameters allowing a more thorough sampling and optimization (exhaustive_scoring (chemgauss3), rstep (0.75), tstep (0.5), num_poses $(10,000)$, clash_scale (0.75), opt (chemgauss3), hitlist_size (1,000,000), num_alt_poses (100), sort_by_top_consensus_pose (true). Consensus pose selection was carried out using the following scoring functions and weights: pose_select_weight_shapegauss 1, pose_select_weight_plp 1, pose_select_weight_chemgauss3 1, pose_select_weight_oechemscore 1, pose_select_weight_screenscore 1. Consensus scoring was applied by combining the following scoring functions: shapegauss, plp, chemgauss3, oechemscore, screenscore. 


\section{Tau Protein Preparation}

The human Tau constructs were expressed in pNG2 vector, a derivative of pET-3a (Merck-Novagen, Darmstadt) in E.coli strain BL21(DE3) (Merck-Novagen, Darmstadt) ${ }^{52}$. The expressed protein was purified from bacterial extract by making use of the heat stability of Tau protein. The cell pellet was resuspended in the boiling-extraction buffer (50 mM MES, $500 \mathrm{mM} \mathrm{NaCl}, 1 \mathrm{mM} \mathrm{MgCl} 2,1 \mathrm{mM}$ EGTA, 5 mM DTT, pH 6.8) complemented with protease inhibitor coctail (1 mM PMSF, $1 \mathrm{mM}$ EDTA, $1 \mathrm{mM}$ EGTA, $1 \mathrm{mM}$ benzamidin, $1 \mu \mathrm{g} / \mathrm{ml}$ leupeptin, $1 \mu \mathrm{g} / \mathrm{mL}$ aprotinin, and $1 \mu \mathrm{g} / \mathrm{ml}$ pepstatin). The cells were disrupted with a French pressure cell and subsequently boiled for $20 \mathrm{~min}$. The soluble extract was isolated by centrifugation, the supernatant was dialyzed against the cation exchange chromatography buffer A (20 mM MES, $50 \mathrm{mM} \mathrm{NaCl}, 1 \mathrm{mM}$ EGTA, $1 \mathrm{mM} \mathrm{MgCl} 2,2 \mathrm{mM}$ DTT, $0.1 \mathrm{mM}$ PMSF, pH 6.8) and loaded on cation exchange SP-Sepharose column (GE Healthcare). The protein was eluted by a linear gradient of cation exchange chromatography buffer B (20 mM MES, 1 M NaCl, 1 mM EGTA, 1 mM $\mathrm{MgCl}_{2}, 2 \mathrm{mM}$ DTT, $0.1 \mathrm{mM}$ PMSF, $\mathrm{pH}$ 6.8). The short Tau constructs representing Tau repeat domains Tau3RD (3 repeats; (M)Q244-S372 lacking V275-S305, comprising 99 residues) were subsequently concentrated and rebuffered in PBS (pH 7.4) on Amicon Ultra-15 device (Millipore, Bedford, MA). Protein concentration was determined by the Bradford assay and the purity of the proteins was analyzed by SDS-PAGE (17\%).

\section{Microscale thermophoresis}

Interactions between methylene blue (MB) and K18 or full-length Tau as well as between MB and glutathione (GSH) were characterized by microscale thermophoresis (MST) on a Monolith NT.115 instrument from Nanotemper as follows. The concentration of the fluorescent MB was kept constant at $100 \mathrm{nM}$ and the concentration of proteins was increased in the samples during the MST experiments from 0 to $1400 \mathrm{nM}$. The proteins were dissolved in $10 \mathrm{mM}$ HEPES, $5 \mathrm{mM} \mathrm{NaCl}, 0.005 \%$ Tween 20 buffer solutions at $\mathrm{pH}=7.4 .5 \mu \mathrm{l}$ of the respective samples were filled in MST capillaries by simple capillary forces. The following stages are recorded for each sample: fluorescence signal before turning the IR laser on, thermophoresis of molecules and back diffusion after switching the laser off. The signal was recorded in all capillaries at varying concentration of the non-fluorescent protein. Samples were incubated at $25^{\circ} \mathrm{C}$ within the capillaries for 30 min prior to running measurements. Assays were conducted at $40 \%$ IR-laser power and MST powers (corresponding to the excitation strength of the LED) of $20 \%$. Any change of thermophoretic properties is observed as a change in fluorescence intensity. The thermophoresis signal is plotted against the ligand concentration to obtain a dose-response curve, from which the binding affinity $\left(\mathrm{K}_{\mathrm{d}}\right)$ can be deduced. 


\section{LC-MS}

Oxidation kinetics of glutathione (GSH) (Sigma, St. Louis, MO, USA) were measured by LC-MS as follows. GSH was dissolved in PBS buffer at a final concentration of $250 \mu \mathrm{M} .4 \mathrm{mM}$ Indoprofen (Sigma, St. Louis, MO, USA) was dissolved in MeCN and added as a control for UV detection. Reduced GSH was detected in the presence or absence of $5 \mathrm{mM} \mathrm{MB}$ after 4, 8, 12, 24, 48 and 144 hours of incubation. Eluent A contained $10 \mathrm{mM}$ ammonium-formate dissolved in 95\% distilled water and 5\% $\mathrm{MeCN}$. Eluent B contained $10 \mathrm{mM}$ ammonium-formate dissolved in $20 \%$ distilled water and $80 \% \mathrm{MeCN}$. The gradient was changed from $0 \%$ to $100 \% \mathrm{~B}$ in 1 min then hold $100 \% \mathrm{~B}$ for $2.5 \mathrm{~min}$ and changed gradually to $0 \% \mathrm{~B}$ in $1.5 \mathrm{~min}$ and hold at $0 \%$ for $0.5 \mathrm{~min}$. The operating conditions were as follows: nebulizer gas: $1.5 \mathrm{~L} / \mathrm{min}$; drying gas: $15 \mathrm{~L} / \mathrm{min}$; temperature of desolvation lines: $300{ }^{\circ} \mathrm{C}$; temperature of column: $30{ }^{\circ} \mathrm{C}$; time of measurement: $5 \mathrm{~min}$; ionization: DUIS; recorded m/z range: 50-1000; column: WATREX rs15.9e.s1003, Reprospher 100 C18, 5 m, 100x3 mm; instrument: Shimadzu LCMS-2020

\section{Statistics}

To assess the significance of the differences between the distributions we applied KolmogorovSmirnov test, which is a nonparametric test to compare two distributions with different sample sizes as well as two sample, independent Student t-tests. Statistical analysis was carried out by XLSTAT ${ }^{53}$.

\section{SUPPLEMENTARY INFORMATION (SI)}

SI contains supplementary material on the structural characterization of Tau conformational ensembles and on the description of the binding sites of MB on monomeric Tau.

\section{AUTHOR INFORMATION}

\section{Corresponding Author}

* Gergely Tóth at toth.gergely@ttk.mta.hu

\section{Present Addresses}

$\uparrow$ Semmelweis University Department of Biophysics and Radiation Biology, Budapest, Hungary

II University of Arizona, College of Medicine, Department of Pharmacology, Tucson, AZ, USA 


\title{
Author Contributions
}

R. K. co-designed in silico modelling and LC-MS studies and co-performed them with G. Cs. K. S. and A. K. performed all MST experiments. M. P. generated recombinant K18 and full length Tau. G. T. conceived the study, co-designed research experiments, analysed data and contributed to writing the manuscript. All other authors contributed to the analyses of the data and to writing the manuscript. All authors have given approval to the final version of the manuscript.

\section{Funding Sources}

We thank the Hungarian Brain Research Program 2017-1.2.1-NKP-2017-00002 for its funding support.

\section{Acknowledgment}

We thank György M. Keserü for his support with molecular modeling. We also thank the Hungarian NIIF for providing the HPC computing facilities.

\section{Competing financial interests}

We have read the journal's policy and make the following statements on competing financial interests: some of the authors are employees and/or shareholders of Cantabio Pharmaceuticals as designated by Cantabio affiliation. This does not alter our adherence to all the Science policies on sharing data and materials.

\begin{abstract}
ABBREVIATIONS
ENS\#1, ensemble 1; ENS\#2, ensemble 2; MB, methylene-blue; AD, Alzheimer's disease; FTD, frontotemporal dementias; NFTs, neurofibrillary tangles; MAPT, microtubule associated protein Tau; IDP, intrinsically disordered proteins; $\mathrm{Rg}$, radii of gyration; MD molecular dynamics; MST, microscale thermophoresis; LCMS, liquid chromatography-mass spectrometry; RMSD, root-mean-square deviation; GSH, glutathione; BSA, bovine serum albumin.
\end{abstract}




\section{REFERENCES}

1. Vieira, M. N.; Forny-Germano, L.; Saraiva, L. M.; Sebollela, A.; Martinez, A. M.; Houzel, J. C.; De Felice, F. G.; Ferreira, S. T., Soluble oligomers from a non-disease related protein mimic Abeta-induced tau hyperphosphorylation and neurodegeneration. J Neurochem 2007, 103 (2), 736-48.

2. Ballatore, C.; Lee, V. M.; Trojanowski, J. Q., Tau-mediated neurodegeneration in Alzheimer's disease and related disorders. Nat Rev Neurosci 2007, 8 (9), 663-72.

3. Olney, N. T.; Spina, S.; Miller, B. L., Frontotemporal Dementia. Neurol Clin 2017, 35 (2), 339 374.

4. $\quad$ Sigurdsson, E. M., Tau Immunotherapy. Neurodegener Dis 2016, 16 (1-2), 34-8.

5. Coughlin, D.; Irwin, D. J., Emerging Diagnostic and Therapeutic Strategies for Tauopathies. Curr Neurol Neurosci Rep 2017, 17 (9), 72.

6. Ozenne, V.; Schneider, R.; Yao, M.; Huang, J. R.; Salmon, L.; Zweckstetter, M.; Jensen, M. R.; Blackledge, M., Mapping the potential energy landscape of intrinsically disordered proteins at amino acid resolution. $J$ Am Chem Soc 2012, 134 (36), 15138-48.

7. Mukrasch, M. D.; Markwick, P.; Biernat, J.; Bergen, M.; Bernado, P.; Griesinger, C.; Mandelkow, E.; Zweckstetter, M.; Blackledge, M., Highly populated turn conformations in natively unfolded tau protein identified from residual dipolar couplings and molecular simulation. $J$ Am Chem Soc 2007, 129 (16), 5235-43.

8. Mukrasch, M. D.; Bibow, S.; Korukottu, J.; Jeganathan, S.; Biernat, J.; Griesinger, C.; Mandelkow, E.; Zweckstetter, M., Structural polymorphism of 441-residue tau at single residue resolution. PLoS Biol 2009, 7 (2), e34.

9. Fischer, D.; Mukrasch, M. D.; von Bergen, M.; Klos-Witkowska, A.; Biernat, J.; Griesinger, C.; Mandelkow, E.; Zweckstetter, M., Structural and microtubule binding properties of tau mutants of frontotemporal dementias. Biochemistry 2007, 46 (10), 2574-82.

10. Varadi, M.; Vranken, W.; Guharoy, M.; Tompa, P., Computational approaches for inferring the functions of intrinsically disordered proteins. Front Mol Biosci 2015, 2, 45.

11. Fisher, C. K.; Huang, A.; Stultz, C. M., Modeling intrinsically disordered proteins with bayesian statistics. J Am Chem Soc 2010, 132 (42), 14919-27.

12. Toth, G.; Gardai, S. J.; Zago, W.; Bertoncini, C. W.; Cremades, N.; Roy, S. L.; Tambe, M. A.; Rochet, J. C.; Galvagnion, C.; Skibinski, G.; Finkbeiner, S.; Bova, M.; Regnstrom, K.; Chiou, S. S.; Johnston, J.; Callaway, K.; Anderson, J. P.; Jobling, M. F.; Buell, A. K.; Yednock, T. A.; Knowles, T. P.; Vendruscolo, M.; Christodoulou, J.; Dobson, C. M.; Schenk, D.; McConlogue, L., Targeting the intrinsically disordered structural ensemble of alpha-synuclein by small molecules as a potential therapeutic strategy for Parkinson's disease. PLoS One 2014, 9 (2), e87133.

13. Zhu, M.; De Simone, A.; Schenk, D.; Toth, G.; Dobson, C. M.; Vendruscolo, M., Identification of small-molecule binding pockets in the soluble monomeric form of the Abeta42 peptide. $J$ Chem Phys 2013, 139 (3), 035101.

14. Zhang, Y.; Cao, H.; Liu, Z., Binding cavities and druggability of intrinsically disordered proteins. Protein Sci 2015, 24 (5), 688-705.

15. Mollica, L.; Bessa, L. M.; Hanoulle, X.; Jensen, M. R.; Blackledge, M.; Schneider, R., Binding Mechanisms of Intrinsically Disordered Proteins: Theory, Simulation, and Experiment. Front Mol Biosci 2016, 3, 52.

16. Yu, C.; Niu, X.; Jin, F.; Liu, Z.; Jin, C.; Lai, L., Structure-based Inhibitor Design for the Intrinsically Disordered Protein c-Myc. Sci Rep 2016, 6, 22298. 
17. Larbig, G.; Pickhardt, M.; Lloyd, D. G.; Schmidt, B.; Mandelkow, E., Screening for inhibitors of tau protein aggregation into Alzheimer paired helical filaments: a ligand based approach results in successful scaffold hopping. Curr Alzheimer Res 2007, 4 (3), 315-23.

18. Bulic, B.; Pickhardt, M.; Schmidt, B.; Mandelkow, E. M.; Waldmann, H.; Mandelkow, E., Development of tau aggregation inhibitors for Alzheimer's disease. Angew Chem Int Ed Engl 2009, 48 (10), 1740-52.

19. Tomasic, T.; Peterlin Masic, L., Rhodanine as a scaffold in drug discovery: a critical review of its biological activities and mechanisms of target modulation. Expert Opin Drug Discov 2012, 7 (7), 549-60.

20. JW, M. N.; Blackburn, S., Quantification of frequent-hitter behavior based on historical highthroughput screening data. Future Med Chem 2014, 6 (10), 1113-26.

21. Baell, J. B.; Holloway, G. A., New substructure filters for removal of pan assay interference compounds (PAINS) from screening libraries and for their exclusion in bioassays. J Med Chem 2010, 53 (7), 2719-40.

22. Pickhardt, M.; Neumann, T.; Schwizer, D.; Callaway, K.; Vendruscolo, M.; Schenk, D.; St George-Hyslop, P.; Mandelkow, E. M.; Dobson, C. M.; McConlogue, L.; Mandelkow, E.; Toth, G., Identification of Small Molecule Inhibitors of Tau Aggregation by Targeting Monomeric Tau As a Potential Therapeutic Approach for Tauopathies. Curr Alzheimer Res 2015, 12 (9), 814-28.

23. Pickhardt, M.; Biernat, J.; Hubschmann, S.; Dennissen, F. J. A.; Timm, T.; Aho, A.; Mandelkow, E. M.; Mandelkow, E., Time course of Tau toxicity and pharmacologic prevention in a cell model of Tauopathy. Neurobiol Aging 2017, 57, 47-63.

24. Schirmer, R. H.; Adler, H.; Pickhardt, M.; Mandelkow, E., "Lest we forget you--methylene blue...". Neurobiol Aging 2011, 32 (12), 2325 e7-16.

25. Wischik, C. M.; Edwards, P. C.; Lai, R. Y.; Roth, M.; Harrington, C. R., Selective inhibition of Alzheimer disease-like tau aggregation by phenothiazines. Proc Natl Acad Sci U S A 1996, 93 (20), 11213-8.

26. Taniguchi, S.; Suzuki, N.; Masuda, M.; Hisanaga, S.; Iwatsubo, T.; Goedert, M.; Hasegawa, M., Inhibition of heparin-induced tau filament formation by phenothiazines, polyphenols, and porphyrins. J Biol Chem 2005, 280 (9), 7614-23.

27. Crowe, A.; James, M. J.; Lee, V. M.; Smith, A. B., 3rd; Trojanowski, J. Q.; Ballatore, C.; Brunden, K. R., Aminothienopyridazines and methylene blue affect Tau fibrillization via cysteine oxidation. J Biol Chem 2013, 288 (16), 11024-37.

28. Akoury, E.; Pickhardt, M.; Gajda, M.; Biernat, J.; Mandelkow, E.; Zweckstetter, M., Mechanistic basis of phenothiazine-driven inhibition of Tau aggregation. Angew Chem Int Ed Engl 2013, 52 (12), 3511-5.

29. Pakavathkumar, P.; Sharma, G.; Kaushal, V.; Foveau, B.; LeBlanc, A. C., Methylene Blue Inhibits Caspases by Oxidation of the Catalytic Cysteine. Sci Rep 2015, 5, 13730.

30. Stack, C.; Jainuddin, S.; Elipenahli, C.; Gerges, M.; Starkova, N.; Starkov, A. A.; Jove, M.; Portero-Otin, M.; Launay, N.; Pujol, A.; Kaidery, N. A.; Thomas, B.; Tampellini, D.; Beal, M. F.; Dumont, M., Methylene blue upregulates Nrf2/ARE genes and prevents tau-related neurotoxicity. Hum Mol Genet 2014, 23 (14), 3716-32.

31. Hochgrafe, K.; Sydow, A.; Matenia, D.; Cadinu, D.; Konen, S.; Petrova, O.; Pickhardt, M.; Goll, P.; Morellini, F.; Mandelkow, E.; Mandelkow, E. M., Preventive methylene blue treatment preserves cognition in mice expressing full-length pro-aggregant human Tau. Acta Neuropathol Commun 2015, 3, 25.

32. Yao, T. M.; Tomoo, K.; Ishida, T.; Hasegawa, H.; Sasaki, M.; Taniguchi, T., Aggregation analysis of the microtubule binding domain in tau protein by spectroscopic methods. $J$ Biochem 2003, 134 (1), 91-9.

33. von Bergen, M.; Friedhoff, P.; Biernat, J.; Heberle, J.; Mandelkow, E. M.; Mandelkow, E., Assembly of tau protein into Alzheimer paired helical filaments depends on a local sequence 
motif ((306)VQIVYK(311)) forming beta structure. Proc Natl Acad Sci U S A 2000, 97 (10), 5129-34.

34. Barghorn, S.; Biernat, J.; Mandelkow, E., Purification of recombinant tau protein and preparation of Alzheimer-paired helical filaments in vitro. Methods Mol Biol 2005, 299, 35-51.

35. Kadavath, H.; Jaremko, M.; Jaremko, L.; Biernat, J.; Mandelkow, E.; Zweckstetter, M., Folding of the Tau Protein on Microtubules. Angew Chem Int Ed Engl 2015, 54 (35), 10347-51.

36. Kozakov, D.; Grove, L. E.; Hall, D. R.; Bohnuud, T.; Mottarella, S. E.; Luo, L.; Xia, B.; Beglov, D.; Vajda, S., The FTMap family of web servers for determining and characterizing ligandbinding hot spots of proteins. Nat Protoc 2015, 10 (5), 733-55.

37. Flanagan, M. E.; Abramite, J. A.; Anderson, D. P.; Aulabaugh, A.; Dahal, U. P.; Gilbert, A. M.; Li, C.; Montgomery, J.; Oppenheimer, S. R.; Ryder, T.; Schuff, B. P.; Uccello, D. P.; Walker, G. S.; Wu, Y.; Brown, M. F.; Chen, J. M.; Hayward, M. M.; Noe, M. C.; Obach, R. S.; Philippe, L.; Shanmugasundaram, V.; Shapiro, M. J.; Starr, J.; Stroh, J.; Che, Y., Chemical and computational methods for the characterization of covalent reactive groups for the prospective design of irreversible inhibitors. J Med Chem 2014, 57 (23), 10072-9.

38. Sormanni, P.; Piovesan, D.; Heller, G. T.; Bonomi, M.; Kukic, P.; Camilloni, C.; Fuxreiter, M.; Dosztanyi, Z.; Pappu, R. V.; Babu, M. M.; Longhi, S.; Tompa, P.; Dunker, A. K.; Uversky, V. N.; Tosatto, S. C.; Vendruscolo, M., Simultaneous quantification of protein order and disorder. Nat Chem Biol 2017, 13 (4), 339-342.

39. Varadi, M.; Kosol, S.; Lebrun, P.; Valentini, E.; Blackledge, M.; Dunker, A. K.; Felli, I. C.; Forman-Kay, J. D.; Kriwacki, R. W.; Pierattelli, R.; Sussman, J.; Svergun, D. I.; Uversky, V. N.; Vendruscolo, M.; Wishart, D.; Wright, P. E.; Tompa, P., pE-DB: a database of structural ensembles of intrinsically disordered and of unfolded proteins. Nucleic Acids Res 2014, 42 (Database issue), D326-35.

40. Schrödinger, Release 2016-1, Schrödinger, New York, NY, USA, 2016.

41. Miao, Z.; Cao, Y.; Jiang, T., RASP: rapid modeling of protein side chain conformations. Bioinformatics 2011, 27 (22), 3117-22.

42. Touw, W. G.; Baakman, C.; Black, J.; te Beek, T. A.; Krieger, E.; Joosten, R. P.; Vriend, G., A series of PDB-related databanks for everyday needs. Nucleic Acids Res 2015, 43 (Database issue), D364-8.

43. Kozakov, D.; Hall, D. R.; Chuang, G. Y.; Cencic, R.; Brenke, R.; Grove, L. E.; Beglov, D.; Pelletier, J.; Whitty, A.; Vajda, S., Structural conservation of druggable hot spots in proteinprotein interfaces. Proc Natl Acad Sci U S A 2011, 108 (33), 13528-33.

44. OMEGA 2.5.1.4, OpenEye Scientific Software: Santa Fe, NM, USA, http://www.eyesopen.com

45. Hawkins, P. C.; Skillman, A. G.; Warren, G. L.; Ellingson, B. A.; Stahl, M. T., Conformer generation with OMEGA: algorithm and validation using high quality structures from the Protein Databank and Cambridge Structural Database. J Chem Inf Model 2010, 50 (4), 572-84.

46. QUACPAC 1.6.3; OpenEye Scientific Software: Santa Fe, NM, USA, http://www.eyesopen.com

47. McGann, M., FRED and HYBRID docking performance on standardized datasets. J Comput Aided Mol Des 2012, 26 (8), 897-906.

48. McGann, M., FRED pose prediction and virtual screening accuracy. J Chem Inf Model 2011, 51 (3), 578-96.

49. Kelley, B. P.; Brown, S. P.; Warren, G. L.; Muchmore, S. W., POSIT: Flexible Shape-Guided Docking For Pose Prediction. J Chem Inf Model 2015, 55 (8), 1771-80.

50. OEDOCKING 3.2.0.2; OpenEye Scientific Software: Santa Fe, NM, USA, http://www.eyesopen.com

51. FRED, 2.2.5: OpenEye Scientific Software: Santa Fe, NM, USA http://www.eyesopen.com

52. Barghorn, S.; Zheng-Fischhofer, Q.; Ackmann, M.; Biernat, J.; von Bergen, M.; Mandelkow, E. M.; Mandelkow, E., Structure, microtubule interactions, and paired helical filament aggregation by tau mutants of frontotemporal dementias. Biochemistry 2000, 39 (38), 11714-21.

53. XLSTAT 19.4.; , Addinsoft: Paris, France, 2017. 


\section{Table of Contents Graphic}
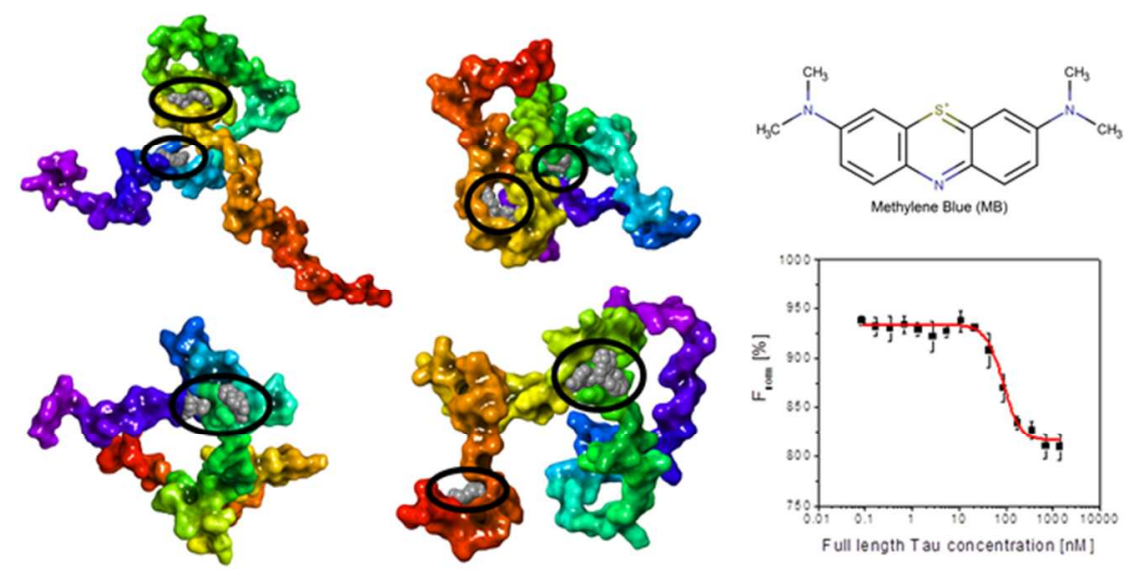